\title{
Catastrophe theory and resonance line shapes in atom-surface scattering
}

\author{
A. L. Glebov, S. Miret-Artés, J. P. Toennies, and F. Traeger \\ Max-Planck-Institut für Strömungsforschung, Bunsenstraße 10, D-37073 Göttingen, Germany \\ J. Margalef-Roig \\ Instituto de Matemáticas y Física Fundamental, Consejo Superior de Investigaciones Científicas, Serrano, 123, 28006 Madrid, Spain
}

(Received 15 January 1999)

\begin{abstract}
The measured high-resolution energy profile of a selective adsorption resonance in the scattering of $\mathrm{He}$ atoms from $\mathrm{NaCl}(001)$ is found to exhibit a mixed-extrema structure reminiscent of a Fano-type function. An analysis of the topological properties of the resonance line shape as a function of the surface temperature reveals a behavior which is isomorphic to the simplest elementary catastrophe, the fold catastrophe. As the crystal temperature approaches the surface Debye temperature $\Theta_{D}$ of $\mathrm{NaCl}$ the resonance profile undergoes a transition in its topology according to the behavior of the fold catastrophe. The analysis is sufficiently general in order to be extended to any resonant scattering event displaying Fano-type profiles.
\end{abstract}

[S0163-1829(99)00633-5]

\section{INTRODUCTION}

Selective adsorption (SA) resonances, first discovered in the $1930 \mathrm{~s}^{1}$, and their line shapes are still perhaps one of the most intriguing phenomena in atom-surface scattering. Several rules governing the intensity features of these resonances have been reported in the literature. ${ }^{2-4}$ The shortcoming of these rules is that they usually require a rather detailed knowledge of the projectile-surface interaction potential and since, in most cases, the potentials are not available, the applicability of these rules is often very difficult and thus limited to only a few systems. However, the different observed resonance peak line shapes or, expressed in mathematical terms, their different topologies, can be understood by a close examination of the individual scattering contributions. ${ }^{5}$

In the present work another theoretical and more general approach is considered for analyzing SA resonance profiles, based on the catastrophe (or singularity) theory (CT) introduced originally by Thom $^{6}$ and later widely developed by Zeeman. ${ }^{7}$ The CT allows a complete description of a system without detailed mathematical treatment of each underlying phenomenon. Taking advantage of its generality it can be applied to many problems in various fields of science, such as physics and physical chemistry, biology, but also psychology or social sciences. Of relevance to this work are the description of light scattering from surfaces ${ }^{8}$ and of caustics and other scattering singularities, of rainbow scattering ${ }^{9,10}$ as well as the description of phase transitions. ${ }^{7}$ The CT provides a comprehensive description of the behavior of the chosen system under variation of external parameters, which is particularly obvious in its applications to phase transitions, e.g., the topology of the Van der Waals equation, which determines the external conditions under which the system adopts a certain state.

In this work an application to a particular SA resonance line shape will be tested. The chosen SA resonance for $\mathrm{He}$ atoms scattered from the $\mathrm{NaCl}(001)$ surface involves the reciprocal-lattice vector $\mathbf{G}=1.58 \AA^{-1}$ and the bound state with binding energy $\epsilon_{2}=-1.5 \mathrm{meV}$. The dependence on the incident-beam energy exhibits a mixed-extrema structure (Fano-type function). First, it will be shown that the mathematical formulation of the measured line shape in terms of the $S$ matrix is isomorphic to the canonical form of the simplest catastrophe, the fold catastrophe. Then, the evolution of the line shape under variation of the surface temperature will be analyzed. As a result, the $\mathrm{CT}$ will be shown to be able to describe all the different topologies of the resonance line shape observed for a wide range of surface temperatures as well as to characterize the background and resonance contributions to the resonance profile.

The analysis is sufficiently general in order to be extended to any resonant scattering event displaying Fano-type profiles.

\section{EXPERIMENTAL}

The experimental apparatus has been described in detail elsewhere. ${ }^{11}$ A nearly monoenergetic ${ }^{4} \mathrm{He}$ atom beam (full width at half maximum: $\Delta \mathrm{v} / \mathrm{v} \sim 1 \% ; \Delta E / E \sim 2 \%$ ) is generated by continuous expansion of pure ${ }^{4} \mathrm{He}$ gas through a nozzle of $10 \mu \mathrm{m}$ in diameter from a stagnation pressure of about 30 bars. Nozzle temperatures between 40 and $150 \mathrm{~K}$ corresponding to incident-beam energies between 8.6 and $32.0 \mathrm{meV}$ were achieved by resistive heating against the cooling of a refrigerator cryostat. The experimental apparatus has a fixed angle geometry in which $\theta_{i}+\theta_{f}=90.1^{\circ}$, with $\theta_{i}$ and $\theta_{f}$ being incident and final scattering angles, respectively. After scattering from the crystal surface the He atoms are detected by a magnetic mass spectrometer, operating in the ion counting mode. The sample is prepared by cleaving a $9 \times 9 \times 7-\mathrm{mm}^{3} \mathrm{NaCl}$ single crystal in situ at $90 \mathrm{~K}$. The resonance line shapes were observed in the measured specular intensity as a function of the incident-beam energy $E_{i}$ at the fixed incident angle for specular reflection $\left(\theta_{i}=45.05^{\circ}\right)$. This was achieved using the technique of drift spectra, in which the incident-beam energy is scanned by increasing or decreasing the nozzle temperature. In the measurements reported here, the nozzle temperature was continuously in- 
creased at a heating rate of $1.3 \mathrm{~K} / \mathrm{min}$ and the accuracy of relative temperature measurement was $\pm 0.05 \mathrm{~K}$. The conversion of nozzle temperature into incident-beam energy was calibrated by measuring the elastic time of flight (TOF) at several nozzle temperatures in the relevant temperature range, the estimated absolute uncertainty in incident energy is less than $2 \%$, but the reproducibility from one spectrum to the next was much better.

\section{EXPERIMENTAL RESULTS}

Figure 1(a) shows a representative drift spectrum from the $\mathrm{NaCl}(001)$ surface measured along the [110] crystallographic direction at a surface temperature of $T_{s}=110 \mathrm{~K}$ in the range of incident energies $E_{i}=8.8-32 \mathrm{meV}$. A superposition of three major contributions determines the shape of the spectrum in Fig. 1(a):

(i) An overall exponential Debye-Waller attenuation of the specular intensity with increasing $E_{i}$ due to enhanced inelastic multiphonon scattering. The decrease of the scattered specular signal $I$ is then given by

$$
I=I_{0} \cdot e^{-2 W},
$$

where the Debye-Waller factor $2 W$ can be written as ${ }^{12}$

$$
2 W=\frac{24\left[E_{i} \cdot \cos ^{2} \theta_{i}+D\right] m}{\theta_{D}^{2} \cdot k_{B} \cdot \mu} \cdot T_{s},
$$

where $E_{i}$ is the incident-beam energy, $\theta_{i}$ the incident angle with respect to the surface normal, $T_{S}$ the surface temperature, $D$ the well depth of the laterally averaged He-surface interaction potential, $k_{B}$ the Boltzmann constant, $\theta_{D}$ the surface Debye temperature, and $m$ and $\mu$ are the masses of the scattering particle and the atoms of the surface unit cell, respectively.

(ii) For a randomly stepped surface, additional intensity oscillations with $E_{i}$ are brought about by changes in the interference conditions for scattering from adjacent terraces of different heights. The energy spacing of these oscillations allows the determination of the step heights. For mono- and diatomic steps on $\mathrm{NaCl}(001)$, the distance between two maxima in the drift spectrum is about 1.58 and $0.79 \AA^{-1}$, respectively. The calculated position of these maxima are indicated by vertical lines in Fig. 1(a), those which are due to monoatomic steps by full lines, maxima resulting from diatomic steps by both dashed and full. Therefore in the energy range shown in Fig. 1(a), two maxima could be expected due to monoatomic steps and four maxima due to diatomic steps. The amplitudes of these oscillations depend on the step density on the surface. ${ }^{13}$

(iii) The rather large corrugation of the $\mathrm{He}-\mathrm{NaCl}(001)$ potential ${ }^{14}$ leads to intense diffraction peaks comparable in intensity to the specular signal (e.g., see Ref. 14), which depend on the incident-beam energy. Specially, the opening up of new diffraction channels with increasing beam energy leads to irregular dips in the intensity as observed in Fig. $1(\mathrm{a})$.

The selective adsorption resonances, of the type investigated in this work, are on a much smaller scale than all of the modulations mentioned above. The kinematic condition for a

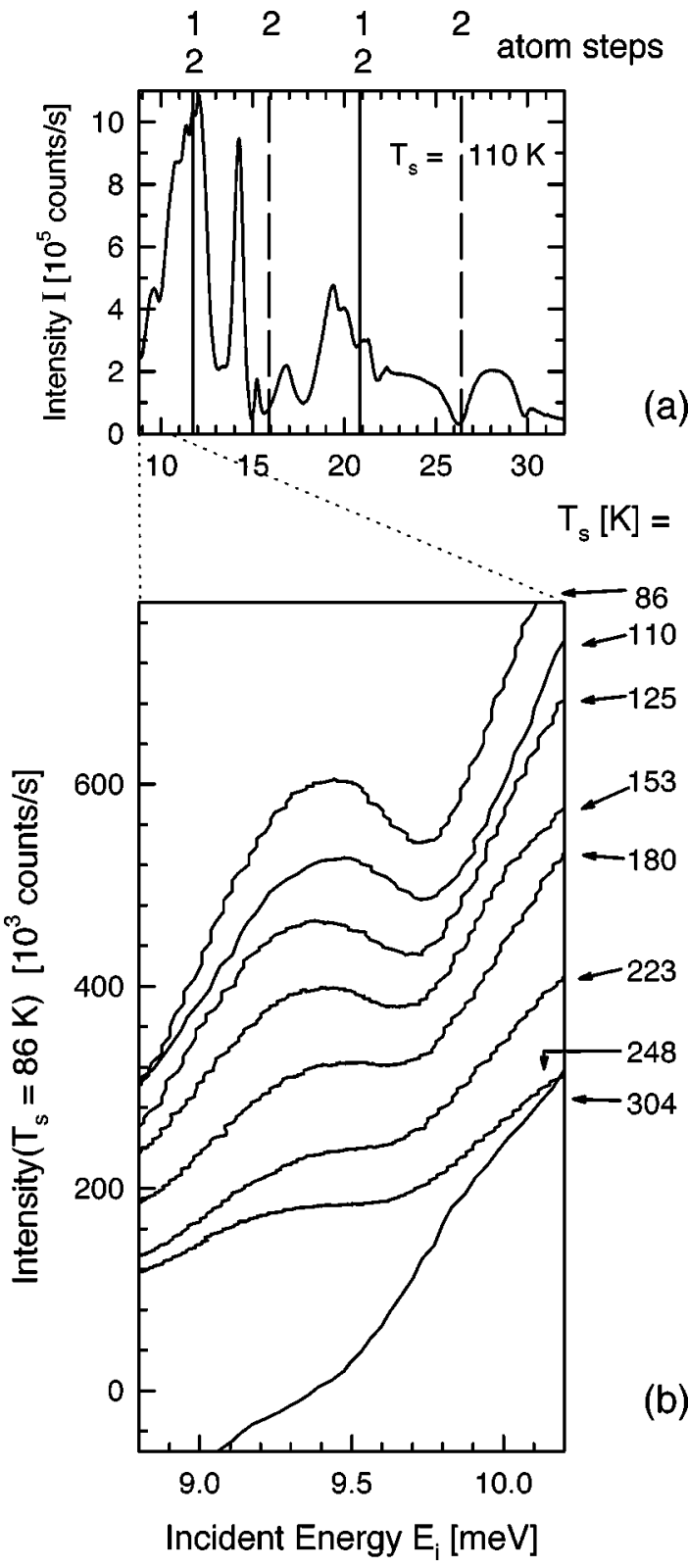

FIG. 1. (a) The specular peak intensity as a function of incident energy $E_{i}$ (drift spectrum) measured at the $\mathrm{NaCl}(001)[110]$ surface for $E_{i}=9-32 \mathrm{meV}$ at a surface temperature of $T_{s}=110 \mathrm{~K}$. Full lines indicate the calculated positions of interference maxima due to mono- and diatomic atomic steps, whereas both dashed and full lines indicate the calculated positions of maxima due to diatomic steps. (b) A series of drift spectra in the range of incident energies $E_{i}=8.8-10.2 \mathrm{meV}$ for eight different surface temperatures between $T_{s}=86$ and $304 \mathrm{~K}$. Only the intensity scale is shown for the drift spectrum for $T_{s}=86 \mathrm{~K}$, the other spectra are plotted to the same scale but shifted for clarity. The resonance observed is due to the coupling to the reciprocal-lattice vector $\mathbf{N}=(1,0) \AA^{-1}$ and the $\mathrm{He}-\mathrm{NaCl}$ bound state $\epsilon_{2}=-1.5 \mathrm{meV}$.

SA resonance assigned to a $n$th bound state of the He-surface interaction potential is given by

$$
-\left|\epsilon_{n}\right|=E_{\mathbf{N} z}=E_{i}-\frac{\hbar^{2}}{2 m}\left[\left(\mathbf{K}_{i}+\mathbf{N}_{\|}\right)^{2}-\mathbf{N}_{\perp}^{2}\right],
$$

where $E_{\mathbf{N} z}$ is the $z$ component of the kinetic energy of the incoming particles, $\mathbf{K}_{i}$ the parallel incident wave vector, and 
$\mathbf{N}=\left(\mathbf{N}_{\|}, \mathbf{N}_{\perp}\right)$ the reciprocal-lattice vector involved in the resonance process, with $\mathbf{N}_{\|}$and $\mathbf{N}_{\perp}$ being the parallel and perpendicular components with respect to the scattering plane, respectively, measured in units of $\mathbf{G}=1.58 \AA-1$.

From Eq. (3) the weak feature with a Fano-type line shape (mixed-extrema, showing both minimum and maximum) observed at $E_{i} \sim 9.5 \mathrm{meV}$ can be assigned to a SA resonance involving the $\epsilon_{2}=-1.5 \mathrm{meV}$ bound state of the ${ }^{4} \mathrm{He}-\mathrm{NaCl}(001)$ interaction potential ${ }^{15}$ and a coupling to the reciprocal-lattice vector $\mathbf{N}=(1,0)$. To investigate this resonance in greater detail drift spectra were measured in the range of incident energies $E_{i}=8.8-10.2 \mathrm{meV}$ near the resonance position at $\bar{E}_{i}=9.5 \mathrm{meV}$ for different surface temperatures $T_{s}$ between 86 and $304 \mathrm{~K}$. Due to the thermal expansion of the crystal the absolute value of the reciprocal-lattice vector $\mathbf{G}=1.58 \AA^{-1}$ varies by about $1 \%$ in this temperature range and according to Eq. (3) will lead to a small shift of the resonance position of about $3 \% .^{16}$ The drift spectra measured at $T_{s} \leqslant 180 \mathrm{~K}$ in Fig. 1(b) show a resonance line shape characteristic of a Fano-type profile with a maximum at $E_{i}$ $=9.4 \mathrm{meV}$ and a minimum at $E_{i}=9.75 \mathrm{meV}$. As discussed below, the topology of this profile changes with $T_{s}$ according to the rules given by the CT.

Fano-type profiles arise from particular interference conditions between the resonance and background scattering contributions, which are mainly due to the short-range attractive and long-range components of the laterally average interaction potential, respectively. The profiles at higher temperatures show a gradual washing out of the resonance while it is hardly visible at $T_{s}=304 \mathrm{~K}$. As seen from Fig. 1(b), with increasing surface temperature, the maximum and minimum of the resonance profile become less pronounced and, finally, form a plateau at a surface temperature of $\sim 248 \mathrm{~K}$. At a higher temperature of $304 \mathrm{~K}$, this plateau is no longer observed, and the specular signal is only slightly modulated around the resonance position. Surprisingly the critical temperature $T_{c}=248 \mathrm{~K}$, where the resonance profile topology changes from the maximum-minimum shape to a plateau, is nearly equal to the surface Debye temperature of $\mathrm{NaCl}$ determined previously to be $\Theta_{D}=250 \mathrm{~K}^{18}$

\section{THEORY}

In this section, first, a theoretical interpretation of the changes of the resonance profile topology as a function of $T_{s}$ is provided using the multichannel resonant scattering theory. ${ }^{19}$ Then, the connection to the CT formalism will be addressed and in the following section a more detailed analysis in terms of CT will be carried out.

In scattering theory, the total probability $P_{t}$ of the transition from the specular channel 0 to a final diffraction channel $g$ via a SA resonance, is given ${ }^{4}$ by the square modulus of the matrix elements of the collision matrix $S$

$$
P_{t}=\left|S_{g 0}\right|^{2}=\left|S_{b, g 0}+i \frac{B_{g 0}}{x+i}\right|^{2},
$$

where $S_{b, g 0}$ is the collision background $S$ matrix, which includes all elastic and inelastic contributions except for those from the SA resonance. Its square modulus gives the probability of the direct, i.e., nonresonant, scattering $P_{b}$
$=\left|S_{b, g 0}\right|^{2}$. A resonant scattering process is described by the matrix element $B_{g 0}$ so that its square modulus $\left|B_{g 0}\right|^{2}=\sigma^{2}$, gives the probabilities of entering $\left(P_{\text {in }}\right)$ and leaving $\left(P_{\text {out }}\right)$ the bound state according to ${ }^{4}$

$$
\sigma^{2}=4 \cdot P_{\text {out }} \cdot P_{\text {in }} .
$$

This square modulus determines the profile of the resonance feature. In general, both probabilities $P_{\text {out }}$ and $P_{i n}$ are equal except when time-invariance no longer holds. ${ }^{19}$ According to the generally accepted independence hypothesis, ${ }^{19}$ these two probabilities $P_{\text {out }}$ and $P_{\text {in }}$ can be treated independently. For convenience, a reduced energy variable $x$, which is a function of the resonance position $\bar{E}_{i}$ and width $\Gamma_{n}$, is introduced: ${ }^{4}$

$$
x=\left(2 / \Gamma_{n}\right) \cdot\left(E_{\mathbf{N} z}-\epsilon_{n}\right) \simeq\left(2 / \gamma_{n, E_{i}}\right) \cdot\left(E_{i}-\bar{E}_{i}\right),
$$

where the width of the resonance $\Gamma_{n}$ is related to the parameter $\gamma_{n, E_{i}}$ through $\Gamma_{n}=\gamma_{n, E_{i}} \cdot\left(\partial E_{\mathbf{N} z} / \partial E_{i}\right)$. Thus $x=0$ gives the resonance position $\bar{E}_{i}$, and $x= \pm 1$ define the borders of the resonance region $\bar{E}_{i} \pm \gamma_{n, E_{i}} / 2$, respectively.

In order to analyze the measured resonance profiles, the experimental data are fitted by an analytical function $F(x)$ which describes the total probability $P_{t}$ defined by Eq. (4) as

$$
F(x)=\alpha+\beta x+\frac{\sigma^{2}+2 \rho(\cos \phi-x \sin \phi)}{x^{2}+1},
$$

where $\quad P_{b}=\left|S_{b, g 0}\right|^{2}=\alpha+\beta x, \quad \sigma^{2}=\left|B_{g 0}\right|^{2}, \quad$ and $\quad \rho^{2}$ $=\left|B_{g 0} \cdot S_{b, g 0}^{*}\right|^{2}$ and hence $b_{r}=\rho \cos \phi$ and $b_{i}=\rho \sin \phi$ are the real and imaginary parts of the product $B_{g 0} \cdot S_{b, g 0}^{*}$, respectively. In obtaining Eq. (7) the assumption is made that the background contribution to the resonance profile is a smooth function of $x$. In particular, the square modulus of $B_{g 0} \cdot S_{b, g 0}^{*}$ is related to the scattering probabilities by the following expression:

$$
\left|B_{g 0} \cdot S_{b, g 0}^{*}\right|^{2}=\rho^{2}=4 \cdot P_{\text {out }} \cdot P_{\text {in }} \cdot P_{b} .
$$

Hereafter, the analytical function $F(x)$ defined by Eq. (7) is used for the topological analysis of SA resonance profiles by means of the CT.

Since SA resonances in drift spectra occur at critical points $x_{0}$ of $F(x)$, determined by the energy of the bound state and the corresponding reciprocal-lattice vector, these points are calculated first. They can be obtained from the condition $F^{\prime}\left(x_{0}\right)=0$, where $F^{\prime}(x)$ stands for the first derivative of the function $F(x)$ with respect to $x$. The type of these critical points is determined by the values of the derivatives of higher orders. If $F^{\prime \prime}\left(x_{0}\right)=0$, the critical point $x_{0}$ is called a twofold degenerate or nonisolated critical point, while for $F^{\prime \prime}\left(x_{0}\right) \neq 0$ it is called nondegenerate and isolated. The appearance of degenerate critical points in a resonance profile when varying some external parameter, such as the substrate temperature, is expected to result in a change in the topology of the resonance profile.

A close examination of the conditions for the critical point gives the first connection to the CT formalism. The background contribution $\left[P_{b}=\alpha+\beta x\right.$ in Eq. (7)], taken as a 
very slowly changing perturbation near the resonance region, affects differently the line shapes of the resonances for isolated and nonisolated critical points. If the linear terms in Eq. (7) are equal to zero $(\beta=0$ and $\sin \phi=0)$, then $x=0$ will never be a twofold degenerate critical point. At these conditions the resonance feature will be reduced to a symmetric Lorentzian function and will show (i) a maximum whenever $P_{b}<P_{\text {out }} \cdot P_{\text {in }}$ and (ii) a minimum whenever $P_{b}>P_{\text {out }} \cdot P_{\text {in }}$. These resonance profiles are structurally stable from a topological point of view. In the case when only $\sin \phi=0$, the resulting resonance profiles are asymmetric but still have either a maximum or a minimum defined by the same conditions. When both linear terms differ from zero, the resonance profiles are given by asymmetric Fano-type mixed-extrema structures. For this case, the resonance position $x=0$ is a twofold degenerate critical point when the following conditions are met: (i) If $F^{\prime}(0)=0$ then $\beta=2 \rho \sin \phi$, that is, the linear terms are equal [see Eq. (7)]. (ii) If $F^{\prime \prime}(0)=0$ then $\cos \phi=-\left(\sigma^{2} / 2 \rho\right)$ which implies that always $P_{b}>P_{\text {out }} \cdot P_{\text {in }}$ and the numerator of Eq. (7) vanishes; (iii) Finally, if $F^{\prime \prime \prime}(0) \neq 0$ then $\sin \phi \neq 0$. These, in fact, are the three necessary conditions for the fold catastrophe. ${ }^{20,21}$ Moreover, it can be shown that the $F(x)$ function is also isomorphic to the fold catastrophe and, therefore, the $F(x)$ function is identical to a canonical function $F(z)$ of the CT (i.e., a simple polynomial of third degree). ${ }^{22}$ Thus these two functions present exactly the same topological properties and can be used to describe exactly the same physical phenomenon.

From CT, it is known ${ }^{20,21}$ that the fold catastrophe has the following topological properties: its bifurcation set is a single point $[a=0$ in the equation for $F(z)$ in Ref. 22] at which a change in the resonance topology occurs. As shown in Fig. 1(b), in the present experiments a change of the SA resonance topology from a maximum-minimum to a plateau is observed at $T_{s}=248 \mathrm{~K}$, giving the critical temperature of $T_{c}=248 \mathrm{~K}$, and therefore the point $x=0$ (the resonance position) can be assigned to a twofold degenerate point. After transforming $F(x)$ to an equivalent function $F(z)$ with a new parameter $a$ as described in a footnote ${ }^{22}$ it follows from the CT that for negative values of $a, F(z)$ and consequently $F(x)$, have two critical points (a maximum and minimum), while for positive $a$ values, no critical point is found in $F(z)$ and $F(x)$. It can be shown that for $a=0$ the relation among the independent parameters of Eq. (7) is given by

$$
\sigma^{2}+2 \rho \cos \phi=(\beta / 3 \sqrt{3}) \cdot(\delta+8) \cdot \sqrt{1-\delta},
$$

with $\delta=2 \rho \sin \phi / \beta \leqslant 1$, which is called the bifurcation set condition. For additional mathematical details see Ref. 22.

\section{APPLICATION OF THE CT THEORY TO THE EXPERIMENTAL DATA}

In order to apply the CT analysis to the experimental data, the resonance profiles shown in Fig. 1(b) have been fitted with the function $F(x)$ from Eq. (7). To do this, the energy scale was converted to the reduced variable $x$ according to Eq. (6). The width of the resonant peak was extracted from the spectra measured at lower surface temperature and fixed at $\gamma_{n, E_{i}}=0.3 \mathrm{meV}$. Equation (7) is shown to be able to describe the different observed line shapes, which, as predicted
TABLE I. Surface temperature $T_{s}$, energetic position $\bar{E}_{i}$ and the parameters $\alpha, \beta, A=\sigma^{2}+2 \rho \cos \phi$, and $B=2 \rho \sin \phi$ from the fitting procedure according to Eq. (7). The width $\gamma_{n, E_{i}}$ was held fixed at $\gamma_{n, E_{i}}=0.3 \mathrm{meV}$. The last column gives the Debye-Waller exponent $2 W$, which is the average number of participating phonons at the present scattering conditions. The drift spectra at $T_{s}=110$ and $304 \mathrm{~K}(*)$ were measured in different runs of the experiment, thus the total intensities are not comparable to the others. A rough extrapolation of the Debye-Waller behavior of $\alpha$ for $T_{s}=304 \mathrm{~K}$ would result in a value of $\alpha \sim 130 \mathrm{kHz}$, if this drift spectrum was taken in the same series as most of the others.

\begin{tabular}{rccrrrr}
\hline \hline$T_{s}[\mathrm{~K}]$ & $\bar{E}_{i}[\mathrm{meV}]$ & $\alpha[\mathrm{kHz}]$ & $\beta[\mathrm{kHz}]$ & $A[\mathrm{kHz}]$ & $B[\mathrm{kHz}]$ & $2 W$ \\
\hline 86 & 9.60 & 601.86 & 9.75 & -11.26 & 75.22 & 0.38 \\
$110^{*}$ & 9.58 & 440.16 & 2.77 & 14.11 & 40.41 & 0.49 \\
125 & 9.52 & 443.29 & 0.54 & 10.61 & 28.89 & 0.55 \\
153 & 9.54 & 392.38 & 10.31 & -3.86 & 35.28 & 0.68 \\
180 & 9.57 & 332.09 & 15.62 & -10.14 & 22.59 & 0.80 \\
223 & 9.52 & 244.34 & 17.05 & -5.70 & 15.71 & 0.99 \\
248 & 9.45 & 183.51 & 8.44 & -0.05 & 8.30 & 1.10 \\
$304 *$ & 9.32 & 557.02 & 34.34 & -8.48 & 12.08 & 1.33 \\
\hline \hline
\end{tabular}

by CT, exhibits a critical point which appears at about $T_{s}=248 \mathrm{~K}$. The experimentally obtained resonance positions $\bar{E}_{i}$ and the four fitting parameters $\alpha, \beta, A=\sigma^{2}+2 \rho \cos \phi$, and $B=2 \rho \sin \phi$ were determined for each drift spectrum of Fig. 1(b). Table I gives these parameters for each fit. Since $\alpha$ and $\beta$ are fitting parameters, the background contribution is fitted for each drift spectrum independently taking into account the fact that the absolute total intensity may differ from run to run of the experiment. This can be seen from Table I for the drift spectrum at $304 \mathrm{~K}$, which was measured in a different run than the others and which shows larger total intensity. Overall, the background should follow a Debye-Waller attenuation, so continuing the series of drift spectra between 86 and $248 \mathrm{~K}$, for $T_{s}=304 \mathrm{~K} \alpha$ can roughly be extrapolated to be $130 \mathrm{kHz}$. This is only mentioned for clarity and does not enter the fit, since, as mentioned above, the background is fitted for each curve independently. More important is the behavior of the fit parameters with respect to each other. The bifurcation set condition Eq. (9) implies that at the critical point $\beta=B$ and $A=0$, which is nearly satisfied at $T_{s}=248 \mathrm{~K}$. For all of the fits a relative error of less than $R=5 \times 10^{-3}$ was estimated according to the equation

$$
R=\frac{1}{N_{p}} \sum \frac{\sqrt{\left(I_{\text {exp }}-I_{f i t}\right)^{2}}}{I_{\text {exp }}},
$$

where $I_{\text {exp }}$ and $I_{\text {fit }}$ are the measured and fitted intensities and $N_{p}$ is the number of points. As an illustration, in Fig. 2, the experimental data (filled circles) and the fitting profiles (solid lines) are plotted for $T_{s}=86,125,180,248$, and $304 \mathrm{~K}$, the background contribution $\alpha+\beta x$ is represented by the dashed lines.

It follows from the CT and it is seen in the data that the change in line shape is accounted for by a critical point. However, since CT describes the behavior of a system by comparing it to certain patterns, the canonical forms, the analysis in terms of the CT is not sufficient to give an inter- 


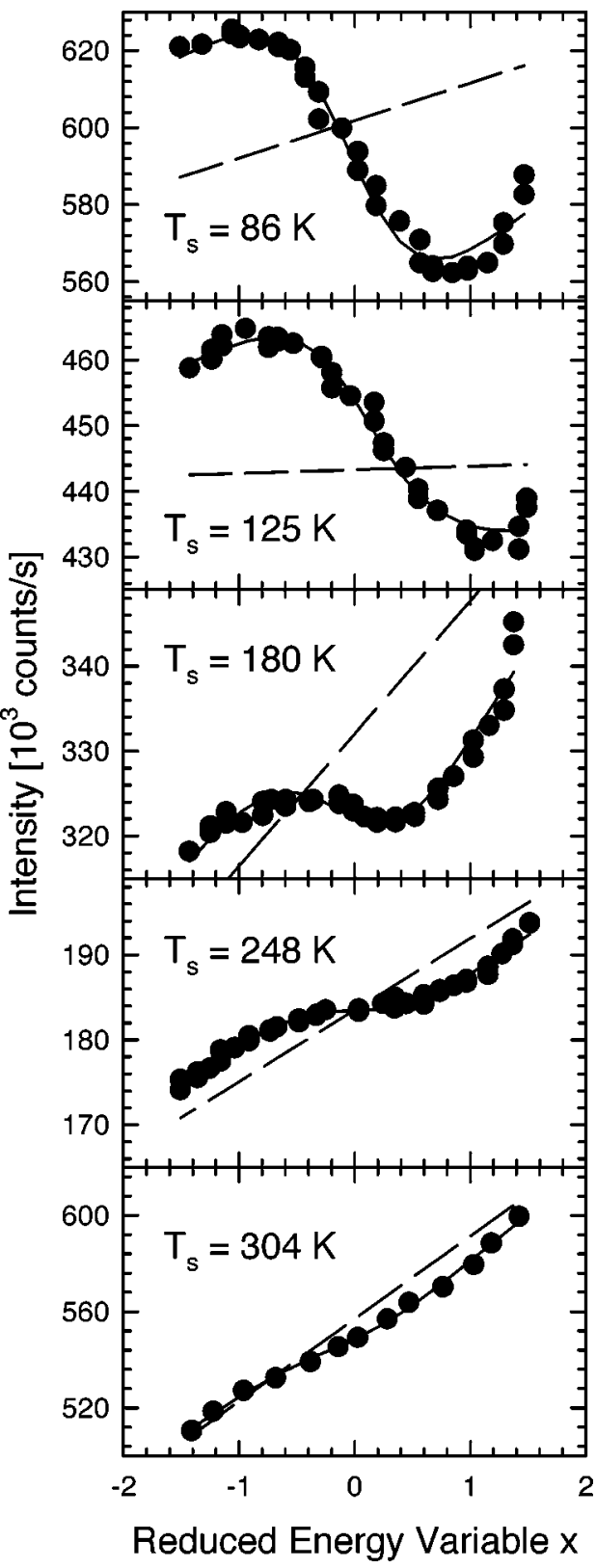

FIG. 2. Examples of the fit of the experimental line shapes (dots) by the $F(x)$ function (solid line) defined by Eq. (7) with the variable $x$ given by Eq. (6). The dashed lines show the linear background term $\alpha+\beta x$. At the surface temperatures $T_{s}=86,125$, and $180 \mathrm{~K}$ two critical points are visible, while at $T_{s}=248 \mathrm{~K}$ only one is visible. At $T_{s}=304 \mathrm{~K}$ no critical points are observed.

pretation of the microscopic phenomena which bring about this critical behavior. As will be discussed below, this change in topology cannot be due to a simple overall DebyeWaller attenuation. Moreover, it is remarkable that the critical temperature $T_{C}$ is very close to the Debye temperature $\Theta_{D}=250 \mathrm{~K}$ of the surface. The value of the Debye-Waller exponent $2 \mathrm{~W}$ can be interpreted as an average number of phonons exchanged in the atom-surface collision process. ${ }^{23}$ The Debye-Waller (DW) exponents for the present experimental conditions at each surface temperature are also listed in Table I. Thus in the case when $2 W \leqslant 1$, single phonon processes are dominant. At the incident-beam energies and surface temperatures used in the present work, contributions of two-phonon and multiphonon processes in inelastic scattering are expected to be very small ( $2 W$ clearly below 2 ). This justifies our assumption that the elastic and inelastic background contributions are smooth functions of $E_{i}$ and therefore are small perturbations to the resonant line shapes. Since the resonance profile studied has a Fano-type shape, an increase of the surface temperature leads to meet the bifurcation condition given by Eq. (9) and the interference between the resonant and background contributions causes a transition in the resonance profile topology. Since CT can no longer be applied when the linear background contribution with $x$ disappears, obviously the corresponding critical temperature should not be much higher than the surface Debye temperature since otherwise the background would no longer be a smooth function of the incident energy $E_{i}$.

In order to have confidence in the above analysis it is necessary to understand the temperature dependence of the resonance profile. The elastic background contribution decreases exponentially with $T_{s}$ according to Eq. (1) and thus the contribution $\alpha+\beta x$ in Eq. (7) is expected to follow an exponential behavior. The parameter $\sigma$, involving the "in" and "out" resonant probabilities, depends only weakly on the temperature since these probabilities are ratios between a partial width (resonance decay by an open diffraction channel) and the total width (resonance decay by all the open diffraction channels $)^{4}$ of the resonance. For elastic SA resonances, these values are only weakly dependent on the surface temperature as well. In contrast, the parameter $\rho^{2}$, expressed in terms of the background probability [see Eq. (8)], follows the thermal DW attenuation and should be the major contribution to the $T_{s}$ dependence of the numerator in Eq. (7). In other words, the cosine and sine functions of $\phi$ are not expected to be very sensitive to the surface temperature. Thus the change of the resonance profile cannot be an artifact resulting from the overall thermal DW attenuation and therefore it must be due to the bifurcation condition given by Eq. (9).

As mentioned above, the CT cannot explain the physical origin behind the transition in the topology, but an analysis in terms of CT reveals the parameters for the critical point. At this stage, it would be desirable to have more experiments in order to confirm the connection with the Debye temperature for other systems. Recently, in the scattering of $\mathrm{D}_{2}$ from $\mathrm{Cu}(001)$ along the [100] direction ${ }^{24}$ a topological transition has also been observed in the so-called resonance "critical" profile at $T_{c}=300 \mathrm{~K}$. This singular profile can been shown to be isomorph to the "cusp" catastrophe and the Debye temperature for this surface has been estimated from He-atom scattering measurements to be $(270 \pm 20) \mathrm{K},{ }^{25}$ which is very close to the critical temperature $T_{c}=300 \mathrm{~K}$. Finally we feel that these two examples indicate that the close correspondence between $T_{c}$ and $\Theta_{D}$ probably is not a coincidence but a result of some underlying physical mechanism.

\section{CONCLUSIONS}

Catastrophe theory has been successfully applied to analyze the SA resonance profiles displaying Fano-type line shapes. The theoretical justification of this analysis was presented along with the experimental investigations of the resonance line-shape topologies for He-atom scattering from 
the $\mathrm{NaCl}(001)$ surface. It was shown that upon the change of some external parameters, such as the surface temperature, the resonance profiles can undergo a topological transition which is isomorphic to the fold catastrophe. Moreover, it was found that for $\mathrm{He}-\mathrm{NaCl}$ surface scattering the catastrophe in the resonance profile topology occurs close to the transition from a single to multiphonon scattering.

The rules governing elastic SA resonance line shapes given in the literature ${ }^{2-4}$ do not take into account the possible degeneracy of the resonance position in terms of some external parameter. In the present study the effect of the surface temperature on the resonance line shapes has been investigated and the $T_{s}$ dependence of the resonance topologies was shown to follow well-defined rules given by the CT.

Finally, we believe that the implications of this resonance profile analysis using the CT could be extended to any scattering event (gas-phase scattering, light scattering, etc.) where Fano-type line shapes are observed. The present theoretical analysis of the resonance profiles has been sufficiently general in order to emphasize its possible implementation to any resonant scattering event.

\section{ACKNOWLEDGMENTS}

This work has been supported in part by DGICYT (Spain) under Contract Nos. PB96-0651-C03-01 and PB95-0071. S.M.A. gratefully acknowledges the Alexander von Humboldt Foundation for financial support. Finally, we would like to thank Professor J. R. Manson and Professor V. Celli for very useful discussions.
${ }^{1}$ R. Frisch and O. Stern, Z. Phys. 84, 430 (1933); J. E. LennardJones and A. F. Devonshire, Nature (London) 137, 1069 (1936); Proc. R. Soc. London, Ser. A 156, 37 (1936).

${ }^{2}$ K. L. Wolfe and J. H. Weare, Phys. Rev. Lett. 41, 1663 (1978).

${ }^{3}$ V. Celli, N. Garcia, and J. Hutchison, Surf. Sci. 87, 112 (1979).

${ }^{4}$ M. Hernández, S. Miret-Artés, P. Villarreal, and G. DelgadoBarrio, Surf. Sci. 274, 21 (1992); S. Miret-Artés, ibid. 294, 141 (1993); 339, 205 (1995).

${ }^{5}$ H. Hoinkes and H. Wilsch, in He Atom Scattering from Surfaces, edited by E. Hulpke (Springer, Berlin, 1992), pp. 113-172, and references therein.

${ }^{6} \mathrm{R}$. Thom, Stabilité Structurelle et Morphogénese (Benjamin, New York, 1972).

${ }^{7}$ E. C. Zeeman, Catastrophe Theory, Selected Papers 1972-1977 (Addison Wesley, Reading, MA, 1977).

${ }^{8}$ M. V. Berry, J. Phys. A 8, 566 (1975).

${ }^{9}$ J. N. L. Connor, Mol. Phys. 31, 33 (1976).

${ }^{10}$ T. Uzer, J. T. Muckerman, and M. S. Child, Mol. Phys. 50, 1215 (1983).

${ }^{11}$ J. P. Toennies and R. Vollmer, Phys. Rev. B 44, 9833 (1991).

12 J. R. Manson, in He Atom Scattering from Surfaces (Ref. 5), pp. 195-200.

${ }^{13}$ B. Flach, E. Hulpke, and W. Steinhögl, Surf. Sci. 412/413, 12 (1998).

${ }^{14}$ L. W. Bruch, A. Glebov, J. P. Toennies, and H. Weiss, J. Chem. Phys. 103, 5109 (1995); G. Drolshagen, A. Kaufhold, and J. P. Toennies, Isr. J. Chem. 22, 283 (1982).

${ }^{15}$ A. Glebov, J. R. Manson, J. G. Skofronick, and J. P. Toennies, Phys. Rev. Lett. 78, 1508 (1997).

${ }^{16}$ The measured thermal expansion of the lattice of about $1 \%$ in the temperature range between 86 and $304 \mathrm{~K}$ is in fairly good agreement with the values of $0.8 \%$ for bulk $\mathrm{NaCl}$ as given in Ref. 17 . Also the overall shift of the resonance position of about $0.3 \mathrm{meV}$ is observed. Deviations between calculated and measured resonance position of about $1 \%$ are found around $T_{s}=200 \mathrm{~K}$, but may be explained by uncertainties in the energy calibration.

${ }^{17}$ Gmelins Handbuch der Anorganischen Chemie, Natrium, 8th ed.,
Suppl. 6 Halogenide (Verlag Chemie, Weinheim, 1973).

${ }^{18}$ A. Glebov, J. P. Toennies, and H. Weiss, Surf. Sci. 351, 200 (1996); L. Ioannotta, G. Scoles, and U. Valbusa, ibid. 161, 411 (1985).

${ }^{19}$ J. R. Taylor, Scattering Theory (Wiley, New York, 1972), p. 411.

${ }^{20}$ T. Poston and I. Stewart, Catastrophe Theory and its Applications (Pitman Publishing, London, 1978).

${ }^{21}$ R. Gilmore, Catastrophe Theory for Scientists and Engineers (Dover Publications, Inc., New York, 1981).

${ }^{22}$ The corresponding algebraic procedure used in this work has been described in J. Gaite, J. Margalef-Roig, and S. Miret-Artés, Phys. Rev. B 57, 13527 (1998); 59, 8593 (1999); J. MargalefRoig and S. Miret-Artés (unpublished). The important point here is to show that the $F(x)$ function and the canonical form for the fold catastrophe are isomorphic. If this is true, it follows that both functions are strictly equal and present the same topological properties as well as describe the same scattering process. Since the mathematical property called transversality (Ref. 7) has been proved to hold, then we can affirm that $F(x)$ is a three-transversal unfolding of a germ which is three determinate. These mathematical concepts can be found in any standard book about the CT. In our context, this result implies that Eq. (7) can explicitly be rewritten as $F(z)=\frac{1}{3} z^{3}+a z+\Pi(\alpha, \beta, \sigma, \rho, \phi)$ after three changes of coordinates are made leading to a new variable $z$ and a perturbation of parameters given by $\Pi$.

${ }^{23}$ J. H. Weare, J. Chem. Phys. 61, 2900 (1974); F. Hofmann, J. P. Toennies, and J. R. Manson, ibid. 106, 1234 (1997).

${ }^{24}$ M. F. Bertino, S. Miret-Artés, and J. P. Toennies, Chem. Phys. Lett. 287, 663 (1998).

${ }^{25}$ F. Hofmann, J. R. Manson, and J. P. Toennies, J. Chem. Phys. 106, 1234 (1997); M. F. Bertino, Ph.D. thesis, University of Göttingen, 1996. A wide range of values for $\Theta_{D}$ between $192 \mathrm{~K}$ (Ref. 26) and $280 \mathrm{~K}$ (Ref. 27) have been reported. For the present experiment the value of $(270 \pm 20) \mathrm{K}$ obtained with HAS in our laboratory appears to be most suitable.

${ }^{26}$ R. J. Reid, Surf. Sci. 29, 623 (1972).

${ }^{27}$ C. Walfried, D. N. Mcllroy, J. Zhang, P. A. Dowben, G. A. Katrich, and E. W. Plummer, Surf. Sci. 363, 296 (1996). 